\title{
Ball-shaped Multiwall Carbon Nanotube Tip by Ion-Beam-Induced Deposition of Platinum
}

\author{
Cheolsu Han ${ }^{1}$, Yung Ho Kahng ${ }^{2}$, In-Yong Park ${ }^{1}$, Byong Chon Park ${ }^{1}$, and Sang Jung Ahn ${ }^{1}$. \\ 1. Center for Advanced Instrumentation, Division of Industrial Metrology, Korea Research Institute of \\ Standards and Science (KRISS), Daejeon 305-340, Republic of Korea \\ 2. Research Institute for Solar and Sustainable Energies, Gwangju 500-712, Republic of Korea
}

Atomic force microscope (AFM) tips with special shapes such as multiwall carbon nanotube (MWNT)attached AFM tips (MWNT tips) [1], shape-modified MWNT tips [2], boot- shaped AFM tips, and ballshaped AFM tips [3] have been introduced. Among these, ball-shaped AFM tip is interesting in critical dimension AFM (CD-AFM) metrology [3], in micro-tribology, and in measurements of the mechanical properties of biological cell. However, conventional ball-shaped tips produced by attaching glass beads are unavailable with nano-scale diameters. Furthermore, it is difficult to control the ball diameter finely or to fabricate nano-scale ball-shaped tips. In this paper, we present how to fabricate a ball-shaped tip by depositing Pt at the free end of a MWNT tip using ion-beam-induced deposition (IBID).

MWNT tip is fabricated by attaching MWNT onto AFM tip using electron beam-induced deposition (EBID) of hydrocarbons. After the MWNT tip is fabricated, we use IBID of Pt to deposit Pt on top of the MWNT tip. The MWNT was first aligned toward $\mathrm{Ga}^{+}$ion beam using the ion-beam-bending process [2] before Pt deposition. During Pt deposition, we supply precursor gas to the sample surface using a gas injection system of dual-beam focused ion beam (FIB, Nova 200, FEI, Co.). The sample surface was saturated with the adsorbed gas molecules. Pt was deposited by ion-energy-induced breaking of the adsorbed precursor within the illuminated area. The ion beam acceleration voltages used were 10, 20, and $30 \mathrm{kV}$, and nominal ion beam currents were 3,23 , and $10 \mathrm{pA}$, respectively. Pt deposition was done in steps, with target thicknesses varying from 10 to $200 \mathrm{~nm}$. According to this predetermined target thickness, the FIB exposed the target area to a programmed amount of Ga ion fluence. We monitored the shape and the lateral size growth of the Pt ball that formed at the free end of the MWNT tip in situ using a scanning electron microscope (SEM).

Figure 1 shows SEM images of Pt ball growth under a $\mathrm{Ga}^{+}$ion beam $(30 \mathrm{kV}, 7.8 \mathrm{pA})$. We observed that the Pt ball grew at the free end of an MWNT tip as the Pt deposition progressed. When the ball size was small, the ball shape was a spheroid (Fig. 1 (b) and (c)). With further deposition, the ball shape gradually transformed into an oblate spheroid (Fig. 1 (d) and (e)). Finally, when the ball size became too big, the ball developed an irregular shape, and this irregular shape persisted (Fig. 1 (f)-(i)). The specifics of the irregular shape development at the final stage of the ball-growing process seemed to be dependent on the growth rate of the $\mathrm{Pt}$ ball. The tube diameter also grew slowly at the initial stage of the deposition process (Fig. 1 (b)- $(\mathrm{g})$ ). However, this growth became faster when the equatorial diameter of the Pt ball exceeded $\sim 350 \mathrm{~nm}$ (Fig. 1 (h) and (i)). Figure 2 shows transmission electron microscope (TEM, 300kV, Tecnai F20S-Twin, FEI, Co.) images of two different Pt ball-shaped tips. We observed that Pt balls formed at the ends of the MWNT tips and that Pt layer coated the sidewalls of the MWNT below. The diameters of the Pt balls and the tubes were measured from the TEM images and an energy dispersive spectrometer (EDS) analysis was also done during TEM analysis. At both locations, Pt nano-particle aggregates were embedded in an amorphous carbon medium, and formation of a continuous Pt metal layer was not observed. The composition of the Pt ball was: $85 \mathrm{~atm} \% \mathrm{C}, 10 \mathrm{~atm} \% \mathrm{Pt}, 3 \mathrm{~atm} \% \mathrm{Si}$, and 2 
atm $\% \mathrm{Ga}$, ignoring the presence of a $\mathrm{Cu}$ signal, which originated from the sample holder.

Since AFM metrology is the most likely potential application of the Pt ball-shaped tips, it is important to know whether a Pt ball-shaped tip is strong enough to withstand the stress of AFM imaging. We did an imaging test with one Pt ball-shaped tip. Both a Pt-coated conventional Si tip and a Pt ball-shaped tip were used to compare scanned images on a patterned Si wafer. The scanned image quality obtained with the Pt ball tip was similar to that obtained with a commercial Pt-coated silicon tip. We observed that the Pt ball was strong enough for the use of AFM imaging from SEM images of the Pt ball-shaped tip before and after the AFM scan.

We report a novel fabrication method to produce nano-ball attached AFM tip using IBID of Pt. The diameter of the ball could be finely controlled up to $\sim 600 \mathrm{~nm}$ and the ball/tube diameter ratio could be adjusted up to $8 \mathrm{~nm}$. Using TEM and EDS analyses, we showed that the attached Pt balls are aggregated Pt nano-particles. We also tested a Pt ball-shaped tip for AFM imaging and found that the Pt ball was strong enough for this application.

\section{References:}

[1] H Dai, et al, Nature 384 (1996) 147.

[2] BC Park, et al, Adv. Mater. 18 (2006) 95.

[3] BC Park, et al, Proc. SPIE 6518 (2007) 651819.

[4] This work was supported by Nano-Materials Technology Development Program through the National Research Foundation of Korea (NRF) funded by the Ministry of Education, Science and Technology (Grant number: 20120009639).
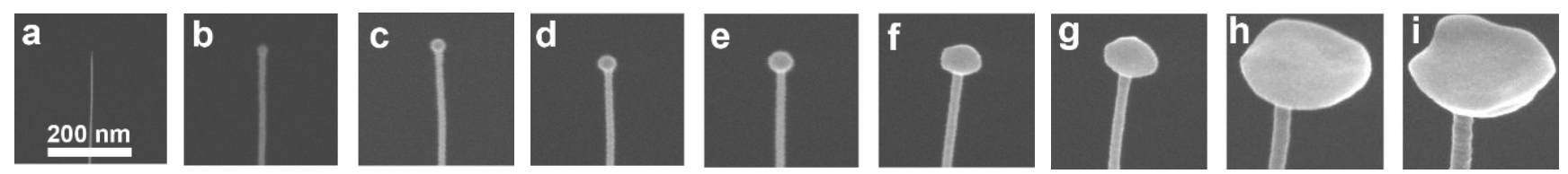

Figure 1. SEM images of Pt ball growth at the free end of a MWNT tip. After the MWNT was aligned by ion beam (a), Pt was deposited in steps with cumulative target thicknesses of: (b) $20 \mathrm{~nm}$, (c) $40 \mathrm{~nm}$, (d) $60 \mathrm{~nm}$ (e) $100 \mathrm{~nm}$, (f) $200 \mathrm{~nm}$, (g) $300 \mathrm{~nm}$, (h) $800 \mathrm{~nm}$, and (i) $1100 \mathrm{~nm}$.
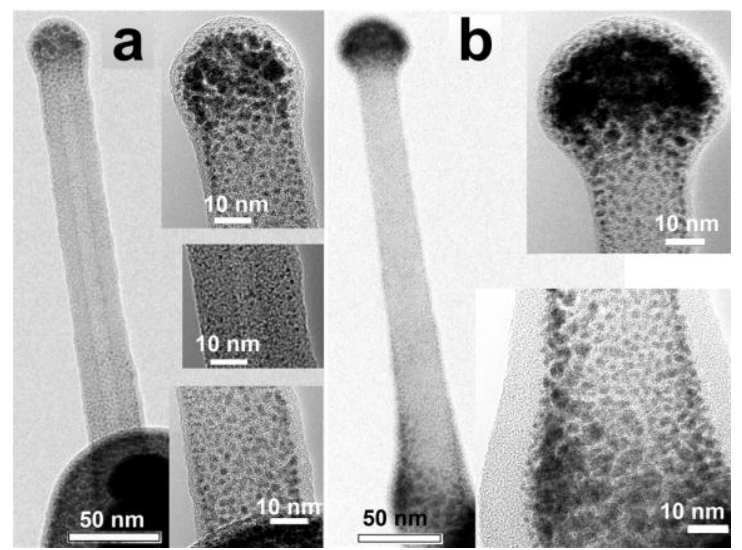

Figure 2. TEM images of Pt ball-shaped tips. Pt deposition target thicknesses were: (a) $10 \mathrm{~nm}$ and (b) $30 \mathrm{~nm}$. Insets show high-resolution TEM images. Pt aggregates (dark spots) existed in the ball as well as on the MWNT. 